Recepción: 13 / 08 / 2018

Aceptación: 09 / 10 / 2018

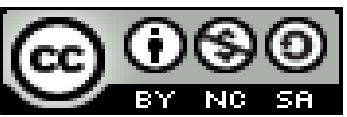

Ciencias del deporte

Publicación: 03 / 12 / 2018

\title{
Aptitud física constructiva. Caso de estudio: Ciudad de Pedernales
}

\section{Constructive physical fitness. Case study: City of Pedernales}

\section{Aptidão física construtiva. Estudo de caso: Cidade de Pedernales}

\author{
Verónica Del R. Suango-Sánchez ${ }^{\text {I }}$ \\ vero_drss@hotmail.com \\ Karen Rodríguez de la Vera ${ }^{\text {II }}$ \\ karen.vera@hotmail.com \\ Víctor J. Moreno-Izquierdo III \\ morenojulio@hotmail.com
}

\author{
Sergio D. Andrade-Sampedro IV \\ s.andrades@hotmail.com \\ Jaime D. Díaz-Tipantiza ${ }^{\vee}$ \\ dariodtipantoza@hotmail.com \\ José A. Yépez-Campoverde VI \\ yepezjose@hotmail.com
}

Correspondencia: vero_drss@hotmail.com

\footnotetext{
${ }^{\text {I }}$ Máster of Science (Msc) Geographical Information Science \& Systems, Ingeniera Geógrafa y del Medio Ambiente, Instituto Espacial Ecuatoriano, Quito, Ecuador.

II Máster Universitario en Tecnologías de la Información Geográfica, Ingeniera Geógrafa y del Medio Ambiente, Instituto Espacial Ecuatoriano, Quito, Ecuador.

III Magíster en Ciencias de la Geoinformación y Observación de la Tierra Mención Información de Tierra para la Planificación del Territorio, Ingeniero Agrónomo, Instituto Espacial Ecuatoriano, Quito, Ecuador.

IV Máster Universitario en Ingeniería Geomántica y Geoinformación, Ingeniero Geógrafo y del Medio Ambiente, Instituto Espacial Ecuatoriano, Quito, Ecuador.

v Máster Universitario en Planificación Territorial y Gestión Ambiental, Ingeniero Agrónomo, Instituto Espacial Ecuatoriano, Quito, Ecuador.

${ }^{V I}$ Máster Universitario en Ingeniería Geodésica y Cartografía, Especialista En Infraestructuras de Datos Espaciales y Su Puesta en Marcha con Herramientas Opensource, Ingeniero Geógrafo y del Medio Ambiente, Instituto Espacial Ecuatoriano, Quito, Ecuador.
} 


\section{Resumen}

El estudio determina la vocación una visión constructiva del territorio, es decir, la Aptitud Física Constructiva (AFC), teniendo como caso de estudio la ciudad de Pedernales en Ecuador; el cual constituye un insumo fundamental para la toma de decisiones acertadas a nivel de Gobiernos Autónomos Descentralizados (GAD), respecto a sus planes de desarrollo, ordenamiento territorial y gestión del riesgo, por ejemplo, ante los impactos que dejó el terremoto del 16 de abril de 2016, aportando significativamente a la planificación urbana. En esta línea, la Teledetección, los Sistemas de Información Geográfica (SIG) y la generación de información geo-espacial son elementos claves que, acompañados de una evaluación multicriterio, permiten la categorización de unidades homogéneas para la compatibilidad e incompatibilidad constructiva en el territorio.

Palabras clave: aptitud constructiva; información geo-espacial; evaluación multicriterio.

\section{Abstract}

The purpose of this research determinates the Constructive Physical Suitability (AFC) in Pedernales. This product constitutes a fundamental input for making sound decisions at the level of Decentralized Autonomous Governments (GAD), with respect to their development plans, land use planning and risk management, for example, given the impact of the earthquake of April 16,2016 , contributing significantly to urban planning. In this line, remote sensing, geographic information systems (GIS) and the generation of geo-spatial information are key elements that, accompanied by multicriteria evaluation, allow the categorization of homogeneous units for compatibility and constructive incompatibility.

Keywords: constructive physical suitability; geo-spatial information; multicriteria evaluation.

\section{Resumo}

O estudo determina a vocação de uma visão construtiva do território, ou seja, a Aptidão Física Construtiva (AFC), tendo como estudo de caso a cidade de Pedernales no Equador; que é um insumo fundamental para a tomada de decisões sólidas no nível dos Governos Autônomos Descentralizados (GAD), com relação aos seus planos de desenvolvimento, planejamento do uso da terra e gerenciamento de risco, por exemplo, antes do impacto do terremoto em 16 de abril de 2016, contribuindo significativamente para o planejamento urbano. Nessa linha, o sensoriamento remoto, os Sistemas de Informações Geográficas (SIG) e a geração de informações geoespaciais 
são elementos-chave que, acompanhados de uma avaliação multicritério, permitem a categorização de unidades homogêneas de compatibilidade e incompatibilidade construtiva no território.

Palavras-chave: aptidão construtiva; informação geoespacial; avaliação multicritério.

\section{Introducción}

Las implicaciones de un desastre son, básicamente, sociales ya que destruye el territorio, mueren personas y, por ende, su estructura social se fractura (Quinceno, 2005).

A partir de la década de los noventa, el mundo ha realizado múltiples esfuerzos para minimizar las vulnerabilidades de las poblaciones afectadas en su nivel social y crecimiento económico, por causa de la materialización de fenómenos adversos, sea estos de origen natural o antrópico. A partir del año 2000 y en adelante, según The United Nations Office for Disaster Risk Reduction (UNISDR, 2012), más de 2,7 billones de personas han sido afectadas: pérdidas económicas alcanzaron 1,3 trillones de dólares y murió 1,1 millón de personas. Por ejemplo, en el año 2010, el terremoto de Haití fue el más mortífero, con más de 220000 víctimas fatales, mientras el terremoto de Chile fue el más costoso en América, con pérdidas que alcanzaron los 30.000 millones de dólares (Emergency Events DatabaseEM-DAT, 2012).

En este contexto, Ecuador también ha sido golpeado por desastres naturales, por encontrarse geográficamente ubicado en la zona de subducción (Instituto Geofísico Escuela Politécnica Nacional IGPN, 2016), tal es el caso del último evento sísmico de magnitud 7,8 Mw en la escala de Richter, ocurrido el pasado 16 abril del 2016, cuyo epicentro estuvo localizado frente a la ciudad de Pedernales, provincia de Manabí, siendo ésta una de las ciudades más afectadas (BBC Mundo, 2016).

Por lo anterior, la ocurrencia de desastres de gran magnitud e impacto deja en evidencia la imposibilidad de eliminar los riesgos a los cuales está sometida la población, y admite las limitaciones de las políticas de prevención ante las vulnerabilidades de la sociedad urbana (Metzger y Robert, 2013). 
La planificación se justifica para evitar los sistemas a funcionales, usos y actividades incompatibles y paisajes amorfos que son el reflejo de una evolución espontánea, la misma que puede involucrar riesgos debido a las amenazas presentes y a la vulnerabilidad existente frente a la misma (Gómez Orea 2002; Cardona, 2008).

En el Ecuador uno de los instrumentos que rigen el ordenamiento territorial es el Código Orgánico de Ordenamiento Territorial, Autonomía y Descentralización (COOTAD) (2015), enmarcado por los principios y reglas generales de la Ley Orgánica de Ordenamiento Territorial, Uso y Gestión de Suelo (LOOTUGS)(2017), que sustenta el deber primordial del Estado para la planificación en pos del desarrollo nacional.

La naturaleza de los desastres que pueden ocurrir en un futuro sugiere que la resiliencia, como concepto, es una visión apropiada para el diseño y planificación de las ciudades, pueblos y resto de comunidades en el futuro (Glavovicy Smith, 2014).

Asimismo, el diseño seguro y económico de la cimentación de las estructuras depende principalmente de la apropiada caracterización del subsuelo (Código Ecuatoriano de la Construcción, 2002). En este sentido, la identificación de espacios propicios para el desarrollo urbano debe respetar las características físicas del suelo y las aptitudes que presente el mismo hacia cualquier actividad.

Por ello, tras la ocurrencia de un desastre, es necesario establecer y definir planes y programas posteriores al evento, donde disponer de información temática a detalle permite tener la capacidad de respuesta para reducir la vulnerabilidad de los sistemas socio-territoriales (Contreras y Beltrán, 2015).

El presente estudio identifica las características físicas del territorio (relieve, suelos, pendientes y litología) de la ciudad de Pedernales, a una escala detallada (1: 5 000), a fin de dar respuesta a la hipótesis: la población de la ciudad de Pedernales se ha asentado en zonas no apropiadas para acoger asentamientos constructivos, por tanto, los mayores daños ocasionados por el terremoto del 16 de abril de 2016 se encuentran en zonas con baja capacidad física para el desarrollo urbano. 


\section{Área de estudio}

El área de interpretación del cantón Pedernales corresponde a 2038 ha, la misma que se encuentra localizada en la provincia de Manabí, al noroeste del Ecuador (Figura 1).Acorde al Censo de Población y Vivienda 201055128 personas viven en el cantón Pedernales (Instituto Nacional de Estadísticas y Censos INEC, 2010), siendo su cabecera cantonal, una de las ciudades más afectadas por el evento sísmico del año 2016 (BBC Mundo, 2016).

Figura 1. Ubicación de la ciudad de Pedernales

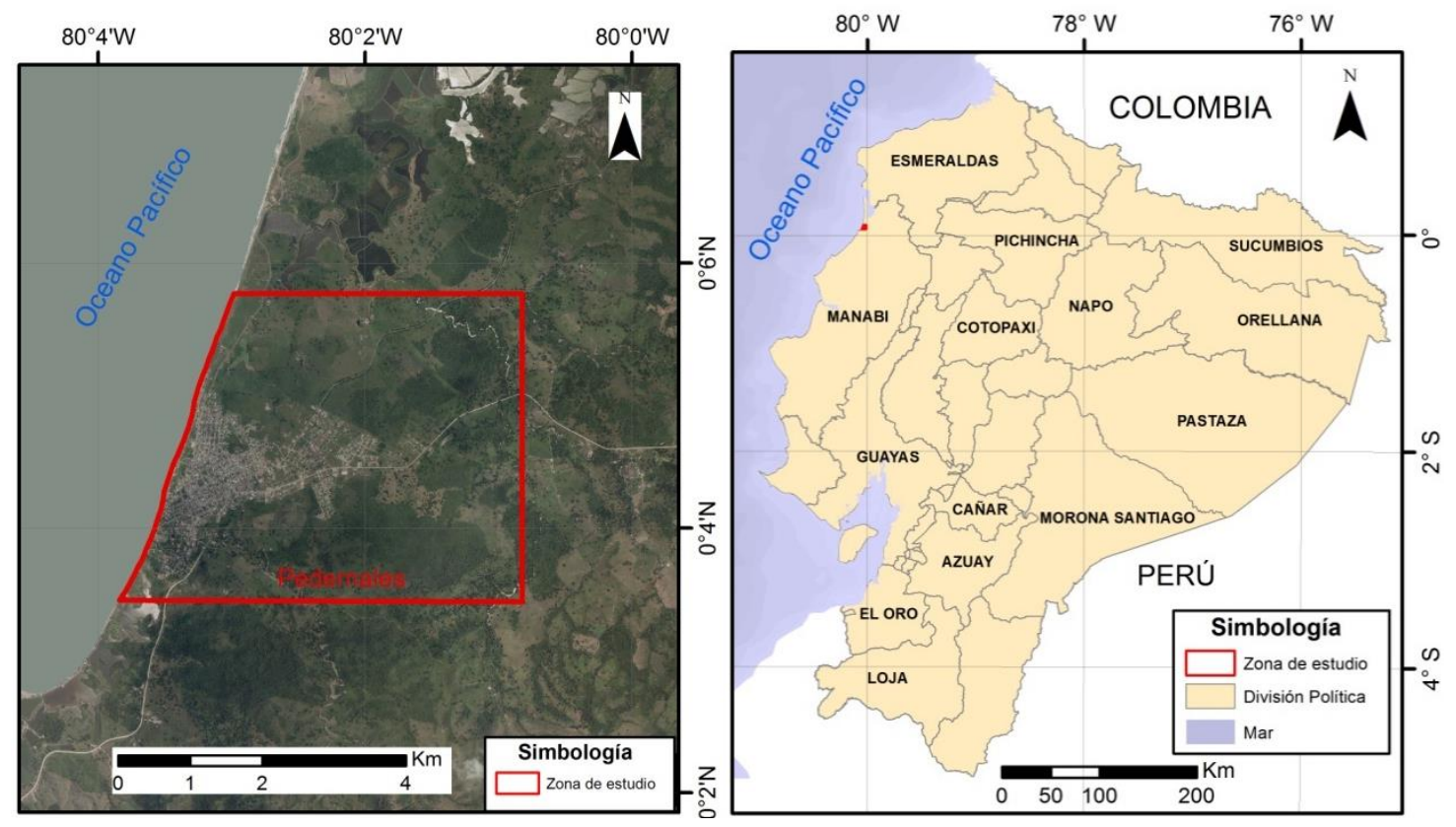

Fuente: Elaboración propia de los autores

\section{Metodología}

De acuerdo a la Figura 2, se inicia con la recopilación de insumos de sensores remotos (ortofotos, imágenes satelitales, modelos digitales de elevación -MDE-) e información secundaria (mapas geológicos, morfopedológicos), que facilitaron la elaboración de información temática primaria a escala detallada (1: 5 000). El análisis espacial integrado, considerando técnicas multicriterio, basado en la ponderación y compensación de variables que influyen de manera positiva (aptitud) o negativa (impacto) (Álvarez, Moreno y Mataix., 2013; Saaty y Vargas, 2013; Yuen, 2013), permitió valorar numéricamente los juicios o criterios dados por expertos. 
Figura 2. Proceso metodológico para la obtención de AFC

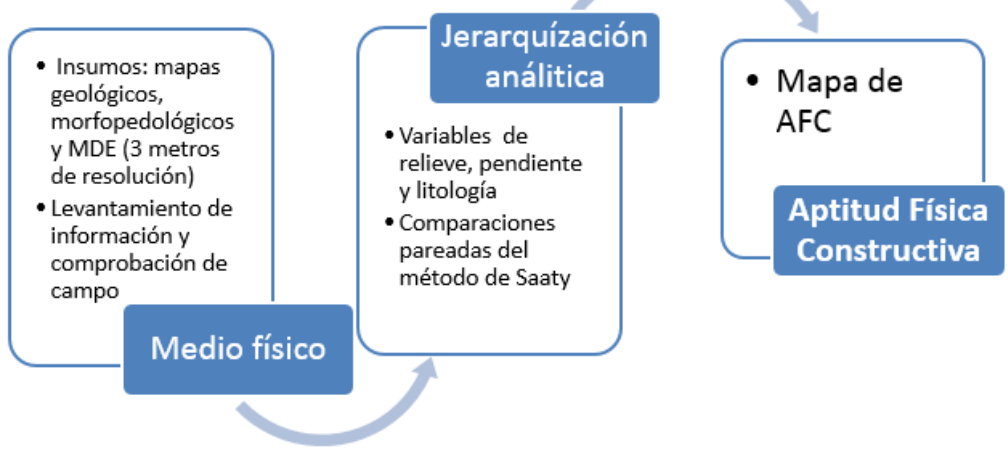

Fuente: Elaboración propia de los autores

Mediante fotointerpretación estereoscópica se delimitan las unidades geomorfológicas a escala 1:5 000, considerando variables como, por ejemplo, unidad ambiental, unidad genética, unidad morfológica, pendiente, tipo de drenaje, densidad de drenaje, geología, litología, depósito superficial(Jumbo, 2018), validadas a través de una visita al campo para, posteriormente, generar el mapa geomorfológico final.

Para obtener la distribución de los suelos en el paisaje se utilizó el enfoque geopedológico (Zinck, 2014; Zinck, Metternicht, Bocco y Del Valle, 2016), permitiendo fragmentar al espacio geográfico a partir de su expresión geomorfológica de acuerdo a sus rasgos homogéneos, caracterizando los suelos mediante la descripción de perfiles (calicatas), obteniendo variables como, por ejemplo, textura, nivel freático, tipo de drenaje, pedregosidad.

Para la determinación de la Aptitud Física Constructiva (AFC) se realiza una calificación en un rango de 1 a 5: relieve y suelos (Tabla 1),pendiente (Tabla 2) y litología (Tabla 3),siendo el valor más alto (5) un indicativo de condiciones altamente desfavorables, por ejemplo, lugares con suelos blandos, de materiales finos/sueltos, pendientes abruptas y nivel freático superficial. 
Tabla 1. Ejemplo de asignación de pesos para el relieve y suelos

\begin{tabular}{|c|c|c|c|}
\hline $\begin{array}{l}\text { UNIDAD } \\
\text { GENÉTICA }\end{array}$ & $\begin{array}{l}\text { UNIDAD } \\
\text { MORFOLÓGICA }\end{array}$ & SUELOS & PESOS \\
\hline Denudativo & Coluvio aluvial antiguo & $\begin{array}{l}\text { Franco arcillo } \\
\text { Limoso }\end{array}$ & 4 \\
\hline \multirow{3}{*}{$\begin{array}{l}\text { Deposicional o } \\
\text { acumulativo }\end{array}$} & Escarpe de terraza & Arcilloso & 5 \\
\hline & Terraza media & Arcilloso & 3 \\
\hline & Terraza media colgada & Arcilloso & 3 \\
\hline \multirow{3}{*}{ Estructural } & Garganta & Arcilloso & 5 \\
\hline & Superficie de mesa & Arcilloso arenoso & 2 \\
\hline & Vertiente de mesa & Arcilloso & 4 \\
\hline \multirow{2}{*}{ Marino y fluviomarino } & Acantilado & Arcilloso arenoso & 5 \\
\hline & Playas emergidas & Arenoso & 5 \\
\hline \multirow[t]{2}{*}{ Formas poligénicas } & $\begin{array}{l}\text { Relieve colinado bajo y } \\
\text { muy bajo }\end{array}$ & Arcilloso & 2 \\
\hline & Relieve colinado medio & Arcilloso & 3 \\
\hline
\end{tabular}

Tabla 2. Asignación de pesos para las pendientes

\begin{tabular}{lc}
\hline \multicolumn{1}{c}{ PENDIENTE \% } & PESOS \\
\hline 0 a 2: casi plana & 1 \\
2 a 5: muy suave & 1 \\
5 a 12: suave & 2 \\
12 a 25: moderada & 3 \\
25 a 40: fuerte & 3 \\
40 a 70: muy fuerte & 4 \\
70 a 100: moderadamente escarpada & 5 \\
> a 100: escarpada & 5 \\
\hline
\end{tabular}


Tabla 3. Ejemplo de asignación de pesos para la litología

\begin{tabular}{lc}
\hline \multicolumn{1}{c}{ GEOLOGÍA/LITOLOGÍA } & PESOS \\
\hline $\begin{array}{l}\text { Depósitos marinos: limos y arenas de grano fino de los depósitos } \\
\text { marinos }\end{array}$ & 5 \\
$\begin{array}{l}\text { Depósitos coluvio aluviales: limos, arcillas, arenas, gravas y bloques } \\
\text { Depósitos aluviales: arcillas, limos y arenas de grano fino a medio }\end{array}$ & 4 \\
$\begin{array}{l}\text { Formación Onzole: limolitas azules (amarillo- café cuando están } \\
\text { meteorizadas) y lutitas con escasas intercalaciones areniscas y }\end{array}$ & 5 \\
$\begin{array}{l}\text { conglomerados } \\
\begin{array}{l}\text { Formación Borbón: areniscas grises-azuladas, de grano medio a } \\
\text { grueso, con lentes conglomeráticos, lamas e intercalaciones locales } \\
\text { de niveles tobáceos y fósiles }\end{array}\end{array}$ \\
\hline
\end{tabular}

Después de la asignación de pesos, se realizó comparaciones pareadas por medio del método de jerarquías analíticas de Saaty (Saaty y Vargas, 2013; Zhang, Tan y Sun, 2015; Gao, Li y Sun, 2018), que permite comparar entre criterios la importancia que tiene el uno sobre el otro, por ejemplo, se le da una calificación de 1 si ambos criterios son de igual importancia hasta el valor de 9 con una importancia absoluta de un criterio sobre otro (Tabla 4).

Tabla 4. Matriz de comparación

\begin{tabular}{lccc}
\hline & $\begin{array}{c}\text { RELIEVE Y } \\
\text { SUELOS }\end{array}$ & PENDIENTE & LITOLOGÍA \\
\hline RELIEVE Y SUELOS & 1 & 4 & $1 / 3$ \\
PENDIENTE & $1 / 4$ & 1 & 3 \\
LITOLOGÍA & 3 & $1 / 3$ & 1 \\
\hline
\end{tabular}

Con la matriz de comparación de criterios se normalizó la matriz, sumando las columnas y dividiendo cada elemento para la suma de la columna, sacando un promedio por fila, dando como resultado los coeficientes de cada variable (Tabla 5). 
Tabla 5. Determinación de coeficientes

\begin{tabular}{|c|c|c|c|c|}
\hline & $\begin{array}{l}\text { RELIEVE Y } \\
\text { SUELOS }\end{array}$ & PENDIENTE & LITOLOGÍA & COEFICIENTES \\
\hline $\begin{array}{l}\text { RELIEVE Y } \\
\text { SUELOS }\end{array}$ & 0,24 & 0,75 & 0,08 & 0,35 \\
\hline PENDIENTE & 0,06 & 0,19 & 0,69 & 0,31 \\
\hline LITOLOGÍA & 0,71 & 0,06 & 0,23 & 0,33 \\
\hline Sumatoria & 1 & 1 & 1 & 1 \\
\hline
\end{tabular}

Con la obtención de los pesos y coeficientes para relieve y suelos, pendiente y litología se calcula la AFC por medio de la fórmula:

$$
\mathrm{AFC}=(\text { Prel } \mathrm{x} \text { Crel })+(\text { Ppen } \mathrm{x} \text { Cpen })+(\text { Plit } \mathrm{x} \text { Clit })
$$

Dónde: Prel: peso del relieve; Crel: coeficiente del relieve; Ppen: peso de pendiente; Cpen: coeficiente de pendiente; Plit: peso de litología; Clit: coeficiente de litología.

Evaluadas las características físicas se llegó a obtener el mapa de distribución de los lugares propicios o no para la implantación de infraestructura urbana bajo cinco niveles: apto para construcción, apto con ligeras limitaciones, apto con moderadas limitaciones, apto con severas limitaciones y no apto para construcción.

Por último, y con oportunidad de colaborar con las tareas de rescate y direccionar el suministro de ayuda en la ciudad de Pedernales, se cuantificó in situ las viviendas colapsadas o con algún tipo de avería por acción de las ondas sísmicas del terremoto del año 2016, las cuales fueron georeferenciadas.

\section{Resultados}

El área de estudio se encuentra dentro de tres unidades ambientales: cordillera costera JamaCoaque, relieves litorales sedimentarios y fluvio marinos, y medio aluvial. Las formaciones localizadas en el área de estudio son dos: Formación Onzole y Formación Borbón. La vía 
Pedernales-Muracungo es aproximadamente el límite entre las dos formaciones, situándose la formación Onzole en dirección Noreste y la Borbón en dirección Noroeste.

Los depósitos superficiales encontrados son tres: a) depósitos coluvio aluviales (distribuidos a lo largo del estero Vaca de Monte y otros drenajes que alimentan al estero Chiquimbo); b) aluviales (localizados a lo largo del río Tachina y el estero Chiquimbo) c) marinos (ubicados en dirección Noreste en todo el perfil costero de la zona de estudio). El origen de las unidades morfológicas corresponden a procesos poligenéticos (relieves colinados), estructurales (superficies disectadas de mesa y vertientes de mesa), denudativos (gargantas y coluvios aluviales antiguos), marino y fluviomarino (acantilados, playas levantadas y playas marinas) y deposicionales (meandros abandonados, terrazas medias, terrazas medias colgadas, escarpes de terraza, terrazas bajas, valles fluviales y cauces actuales).

Los suelos se encuentran influidos por un solo régimen de temperatura isohipertérmico, además de estar, en forma general, en la transición údico-ústico, respecto al régimen de humedad. Ocupando la mayor superficie del territorio se encuentran las texturas superficiales arcillosas $(83,17 \%)$ y arcillo arenosas $(14,73 \%)$, y en menor área se encuentran los suelos de texturas franco arcillo limosas y arenosas con $1,21 \%$ y $0,89 \%$, respectivamente. El drenaje de los suelos es su mayoría son moderados $(87,81 \%)$, con profundidades efectivas, principalmente, poco profundos $(89,42 \%)$ (Tabla 6$)$.

Tabla 6. Características de las unidades geopedológicas

\begin{tabular}{|c|c|c|c|c|c|}
\hline \multicolumn{6}{|c|}{ Descripción de unidades geopedológicas } \\
\hline $\begin{array}{l}\text { Geología/Depósitos } \\
\text { Superficiales }\end{array}$ & Genética & Geoforma & Características & ha & $\%$ \\
\hline \multirow[t]{2}{*}{$\begin{array}{l}\text { Formación Onzole } \\
\text { (Mioceno Superior) }\end{array}$} & \multirow[t]{2}{*}{ Estructural } & $\begin{array}{l}\text { Superficies de } \\
\text { mesa }\end{array}$ & $\begin{array}{l}\text { Corresponden a geoformas } \\
\text { con cimas redondeadas; } \\
\text { con pendientes que van de } \\
2 \text { a } 5 \% \text {, donde los suelos } \\
\text { son de textura arcillosa, } \\
\text { con buen drenaje y } \\
\text { moderadamente profundos } \\
(50 \text { a } 100 \mathrm{~cm}) .\end{array}$ & 285,52 & 14,01 \\
\hline & & $\begin{array}{l}\text { Vertientes de } \\
\text { mesa }\end{array}$ & $\begin{array}{l}\text { Las formas de sus } \\
\text { vertientes son mixtas; con } \\
\text { rangos de pendientes que } \\
\text { van de } 12 \text { a } 40 \% \text {, de } \\
\text { textura arcillo arenosa en la } \\
\text { superficie y arcillosa a }\end{array}$ & 58,05 & 2,85 \\
\hline
\end{tabular}




\begin{tabular}{|c|c|c|c|c|c|}
\hline \multicolumn{6}{|c|}{ Descripción de unidades geopedológicas } \\
\hline $\begin{array}{l}\text { Geología/Depósitos } \\
\text { Superficiales }\end{array}$ & Genética & Geoforma & Características & ha & $\%$ \\
\hline & & & $\begin{array}{l}\text { profundidad, de drenaje } \\
\text { moderado y con una } \\
\text { profundidad efectiva entre } \\
20 \text { a } 50 \mathrm{~cm} \text {. }\end{array}$ & & \\
\hline & & Gargantas & $\begin{array}{l}\text { Sus vertientes tienen } \\
\text { formas irregulares; tienen } \\
\text { un rango de pendientes que } \\
\text { va de } 12 \text { a } 70 \% \text {, texturas } \\
\text { arcillosas en todo el perfil, } \\
\text { con buen drenaje y con } \\
\text { profundidad efectiva entre } \\
20 \text { a } 50 \mathrm{~cm} \text {. }\end{array}$ & 124,16 & 6,09 \\
\hline \multirow{3}{*}{ Formación Borbón } & \multirow{3}{*}{ Poligenético } & $\begin{array}{l}\text { Relieves } \\
\text { colinados } \\
\text { medios }\end{array}$ & $\begin{array}{l}\text { Corresponden a geoformas } \\
\text { con cimas redondeadas, } \\
\text { vertientes irregulares y } \\
\text { mixtas; con pendientes } \\
\text { entre } 25 \text { a } 40 \% \text { (medias a } \\
\text { fuertes) y entre } 40 \text { a } 70 \% \\
\text { (fuertes), con desniveles } \\
\text { relativos entre } 25 \text { a } 50 \mathrm{~m} \text {. }\end{array}$ & 755,55 & 38,05 \\
\hline & & $\begin{array}{l}\text { Relieves } \\
\text { colinados } \\
\text { bajos }\end{array}$ & 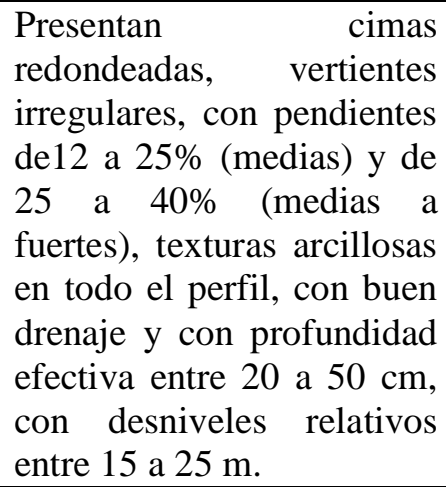 & 331,7 & 16,28 \\
\hline & & $\begin{array}{l}\text { Relieves } \\
\text { colinados muy } \\
\text { bajos }\end{array}$ & $\begin{array}{l}\text { Presentan cimas } \\
\text { redondeadas, con vertientes } \\
\text { irregulares y convexas, con } \\
\text { pendientes de } 5 \text { a } 12 \% \\
\text { (suaves) y de } 12 \text { a } 25 \% \\
\text { (medias), con desniveles } \\
\text { relativos entre } 5 \text { a } 15 \% \text { m, } \\
\text { con drenaje moderado poco } \\
\text { profundos y textura } \\
\text { arcillosa. }\end{array}$ & 199,52 & 9,79 \\
\hline $\begin{array}{l}\text { Depósitos coluvio } \\
\text { aluviales }\end{array}$ & Denudativo & $\begin{array}{l}\text { Coluvio } \\
\text { aluviales } \\
\text { antiguos }\end{array}$ & $\begin{array}{l}\text { Pendientes que van de } 2 \text { a } \\
12 \% \text { y desniveles relativos } \\
\text { entre } 5 \text { a } 15 \quad \mathrm{~m} . \\
\text { Encontrando } \\
\text { arcillosos con }\end{array}$ & 34,79 & 1,71 \\
\hline
\end{tabular}




\section{Descripción de unidades geopedológicas}

\begin{tabular}{|c|c|c|c|c|c|}
\hline $\begin{array}{l}\text { Geología/Depósitos } \\
\text { Superficiales }\end{array}$ & Genética & Geoforma & Características & ha & $\%$ \\
\hline & & & moderado. & & \\
\hline \multirow{3}{*}{ Depósitos marinos } & \multirow{3}{*}{$\begin{array}{l}\text { Marino y } \\
\text { fluvio marino }\end{array}$} & Acantilados & $\begin{array}{l}\text { Presentan vertientes de } \\
\text { forma rectilínea; cuyas } \\
\text { pendientes van de } 70 \text { a } 100 \\
\% \text {, desniveles relativos } \\
\text { entre } 5 \text { a } 25 \text { m y longitudes } \\
\text { de vertientes menores a } 50 \\
\text { m, los cuales son muy } \\
\text { superficiales }(0 \text { a } 10 \mathrm{~cm}) .\end{array}$ & 14,69 & 0,72 \\
\hline & & $\begin{array}{l}\text { Playa } \\
\text { levantada }\end{array}$ & $\begin{array}{l}\text { Se ubica al Noroeste del } \\
\text { área de estudio, cerca de la } \\
\text { vía Pedernales-Cojimíes. } \\
\text { Presenta rangos de } \\
\text { pendientes que van de } 12 \text { a } \\
25 \% \text {. }\end{array}$ & 0,36 & 0,02 \\
\hline & & $\begin{array}{l}\text { Playas } \\
\text { marinas }\end{array}$ & $\begin{array}{l}\text { Cubren el borde Oeste del } \\
\text { área de estudio, frente al } \\
\text { malecón de Pedernales. } \\
\text { Tienen un rango de } \\
\text { pendientes que va de } 0 \text { a } 5 \\
\% \text {. }\end{array}$ & 17,73 & 0,87 \\
\hline \multirow{5}{*}{ Depósitos aluviales } & \multirow{5}{*}{$\begin{array}{l}\text { Deposicional o } \\
\text { acumulativo }\end{array}$} & $\begin{array}{l}\text { Meandro } \\
\text { abandonado }\end{array}$ & $\begin{array}{l}\text { Presenta pendientes que } \\
\text { van de } 0 \text { a } 2 \% \text { y desniveles } \\
\text { relativos entre } 0 \text { a } 5 \mathrm{~m} .\end{array}$ & 0,62 & 0,03 \\
\hline & & $\begin{array}{l}\text { Terrazas } \\
\text { medias }\end{array}$ & $\begin{array}{l}\text { Con pendientes que van de } \\
2 \text { a } 5 \% \text { y desniveles } \\
\text { relativos entre } 0 \text { a } 5 \mathrm{~m} \text {, de } \\
\text { colores negros, arcillosos, } \\
\text { con drenaje de moderado a } \\
\text { mal drenado, y poco } \\
\text { profundos }(20 \text { a } 50 \mathrm{~cm}) .\end{array}$ & 0,19 & 0,01 \\
\hline & & $\begin{array}{l}\text { Terrazas } \\
\text { medias } \\
\text { colgadas } \\
\end{array}$ & $\begin{array}{l}\text { Presentan pendientes que } \\
\text { van de } 2 \text { a } 5 \% \text { y desniveles } \\
\text { relativos entre } 0 \text { a } 5 \mathrm{~m} \text {. }\end{array}$ & 75,58 & 3,51 \\
\hline & & $\begin{array}{l}\text { Escarpes de } \\
\text { terrazas }\end{array}$ & $\begin{array}{l}\text { Se localizan en el río } \\
\text { Tachina. La forma de sus } \\
\text { vertientes es rectilínea; con } \\
\text { pendientes de } 70 \text { a } 100 \% \text {, } \\
\text { desniveles relativos entre } 5 \\
\text { a } 15 \mathrm{~m} \text { y longitudes de } \\
\text { vertientes menores a } 15 \mathrm{~m} \text {. }\end{array}$ & 0,3 & 0,02 \\
\hline & & Terrazas bajas & $\begin{array}{l}\text { Con pendientes que van de } \\
2 \text { a } 5 \% \text { y desniveles } \\
\text { relativos entre } 0 \text { a } 5 \mathrm{~m} \text {, } \\
\text { caracterizados por poseer } \\
\text { texturas arcillosas y colores }\end{array}$ & 30,88 & 1,52 \\
\hline
\end{tabular}




\begin{tabular}{|c|c|c|c|c|c|}
\hline \multicolumn{6}{|c|}{ Descripción de unidades geopedológicas } \\
\hline $\begin{array}{l}\text { Geología/Depósitos } \\
\text { Superficiales }\end{array}$ & Genética & Geoforma & Características & ha & $\%$ \\
\hline & & & $\begin{array}{l}\text { pardo oliva -colores que } \\
\text { reflejan la presencia del } \\
\text { nivel freático desde la } \\
\text { superficie. }\end{array}$ & & \\
\hline & & $\begin{array}{l}\text { Valles } \\
\text { fluviales }\end{array}$ & $\begin{array}{l}\text { Presenta rangos de } \\
\text { pendientes que van de } 2 \text { a } 5 \\
\% \text { y desniveles relativos } \\
\text { entre } 0 \text { a } 5 \mathrm{~m} \text {, cuyos suelos } \\
\text { son arcillosos con mal } \\
\text { drenaje, lo que refleja que } \\
\text { sus colores sean grises } \\
\text { verduzcos muy oscuros. }\end{array}$ & 22,97 & 1,13 \\
\hline & & $\begin{array}{l}\text { Cauces } \\
\text { actuales }\end{array}$ & $\begin{array}{l}\text { Ubicados al Noreste, } \\
\text { Sureste y Suroeste del área } \\
\text { de estudio, principalmente } \\
\text { en el río Tachina, } \\
\text { Presentan rangos de } \\
\text { pendientes que van de } 2 \text { a } 5 \\
\% \text { y desniveles relativos } \\
\text { entre } 0 \text { a } 5 \mathrm{~m} \text {. }\end{array}$ & 53,70 & 2,64 \\
\hline
\end{tabular}

Las geoformas consideradas como no aplicables corresponden a zonas de embalses con 11,74 ha, representando el $0,58 \%$ del área de estudio

\section{Análisis de la Aptitud Física Constructiva (AFC)}

Las áreas con aptitud para la construcción revelan a las superficies de mesa (al Oeste del área de estudio) como las de mayor vocación para la edificación urbana. Las áreas con ligeras limitaciones deben precautelarse en los límites entre otros relieves y en cambios de pendiente. Si existen dificultades de construcción y por ende los costes económicos aumentan paulatinamente en las categorías de aptitud con moderas y severas limitaciones. Las áreas donde se deben prohibir asentamientos son los cuerpos hidrográficos, cauces actuales, playas marinas, terrazas bajas y suelos hidromórficos, especialmente los localizados en el fondo de gargantas o encañonamientos, que constituyen áreas no aptas para construcción debido a que estas zonas pueden ser afectadas por la ocurrencia de amenazas naturales como deslizamientos, flujos de lodo, inundaciones, tsunamis, erosión costera y sismos. El edificar sobre estos espacios serán motivo de creación y aumento de vulnerabilidades y riesgos para la población y sus bienes (Rodríguez, 2017). 
En la Tabla 7 y Figura 3 se detallan la distribución y porcentaje de ocupación de cada uno de los cinco niveles de AFC en los cuales se clasificó el territorio en estudio, donde los niveles con severas limitaciones y no apto para la construcción ocupan el mayor porcentaje del territorio.

Tabla 7. Descripción de AFC en Pedernales

Aptitud

Apto para construcción

limitaciones

Apto con moderadas limitaciones

\section{Distribución espacial} (\% ocupado)

Representan el 14.01\%. Están localizados hacia el Noroeste de la ciudad de Pedernales y corresponden a superficies de mesa, pendientes muy suaves (2-5\%) y materiales de grano medio a grueso, lo cual proporciona mejores condiciones para levantar una edificación o vivienda. Cabe mencionar que, pese a que Pedernales dispone de espacios con aptitud constructiva, no quiere decir que no estén sometidos al impacto de amenaza sísmica, pues este tipo de amenaza es de carácter regional.

Representan el 3.72\%. Ubicados hacia el Suroeste de la ciudad, en las cercanías del río Tachina (terrazas medias colgadas de pendientes muy suaves, con material fino a medio). Estos lugares corresponden a los medios aluviales de origen deposicional o acumulativo. Las construcciones deberán obedecer a las partes más anchas y evitar la edificación cercana a los límites con otras categorías de aptitud. Las amenazas que pueden impactar a estas zonas son los sismos y en algunos casos inundaciones por desbordamiento o anegamiento.

Representan el 9.72\%. Ubicadas hacia el Noreste de la zona con ligeras limitaciones. Se encuentran en esta categoría las unidades de coluvio aluviales antiguos, los relieves colinados bajos y muy bajos conformados por Limolitas azules (amarillo- café cuando están meteorizadas) y lutitas con intercalaciones de areniscas y conglomerados de la Formación Onzole, también están las vertientes de mesa con pendientes hasta el $40 \%$, correspondiente a la formación Borbón.

Representan el 37.78\%. Ubicadas al Noreste de la ciudad. A esta categoría pertenecen las gargantas $\mathrm{y}$, en su mayoría, los relieves colinados medios, de cima redondeada con vertientes irregulares y fuertes pendientes, de la Formación Onzole, que presenta una pendiente máxima del $40 \%$.

Apto con severas limitaciones

Representan el 34.19\%. Ubicadas hacia el Suroeste de la ciudad de

No apto para Pedernales, principalmente. Los terrenos no son aptos para construcción asentamientos urbanos debido a sus características de pendientes fuertes (superiores al 40\%), relieves colinados medios y material fino. 
Figura 3. Aptitud Física Constructiva de Pedernales
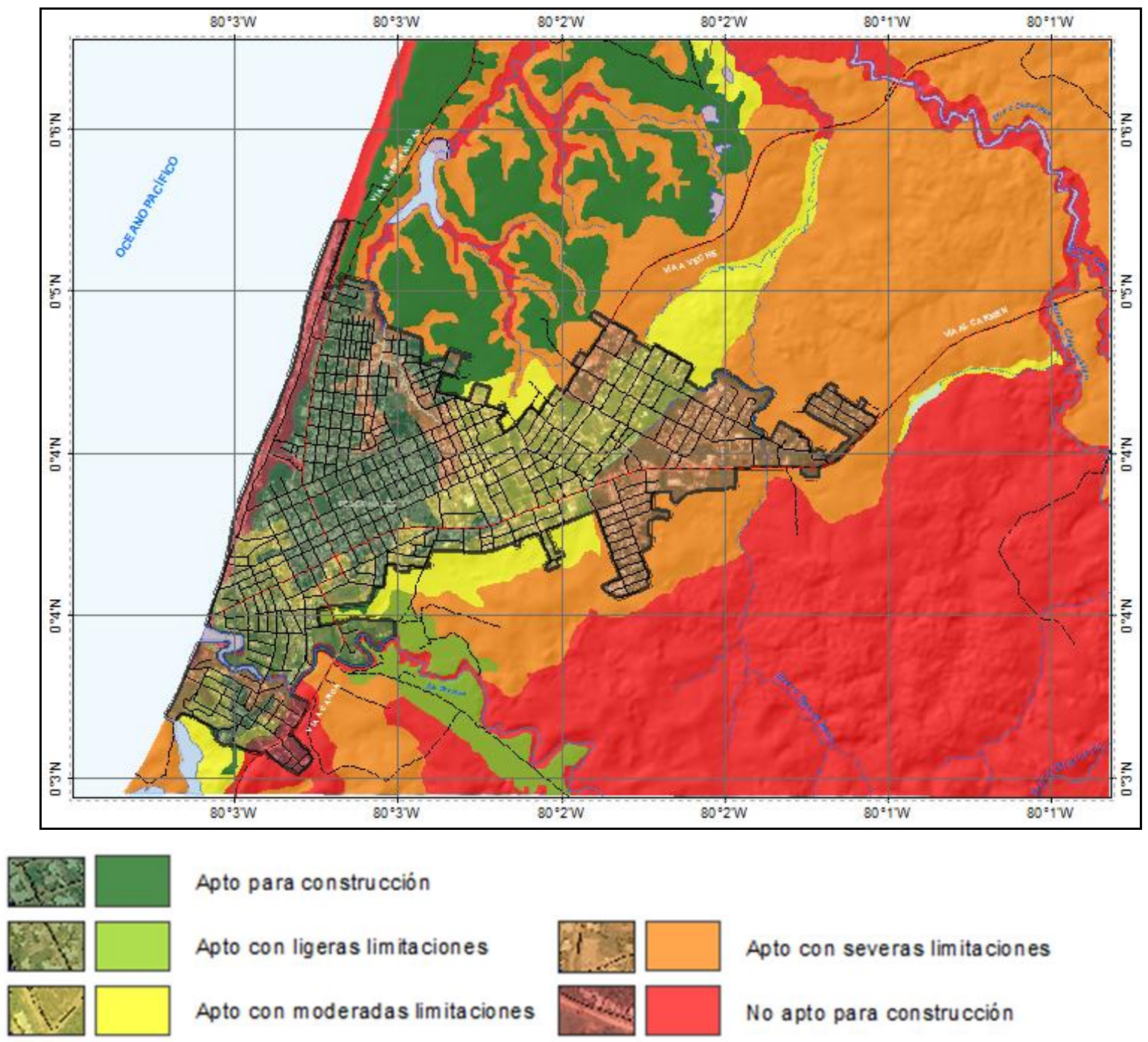

Apto para construcción

Apto con ligeras limitaciones
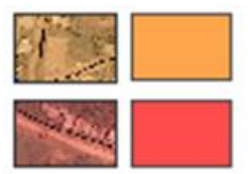

Apto con severas limitaciones

Apto con moderadas limitaciones

Fuente: Elaboración propia de los autores

Con oportunidad de colaborar con las tareas de rescate y direccionar el suministro de ayuda en la ciudad de Pedernales, se comprobó y geo-referenció in situla existencia de 668 viviendas colapsadas o con algún tipo de avería por acción de las ondas sísmicas del terremoto del año 2016. De este total, el 62\% de la infraestructura habitacional presentaba daños y el $38 \%$ fue destruido (Figura 4). 
Figura 4. Tendencia de datos de construcciones afectadas o destruidas en el terremoto, Pedernales

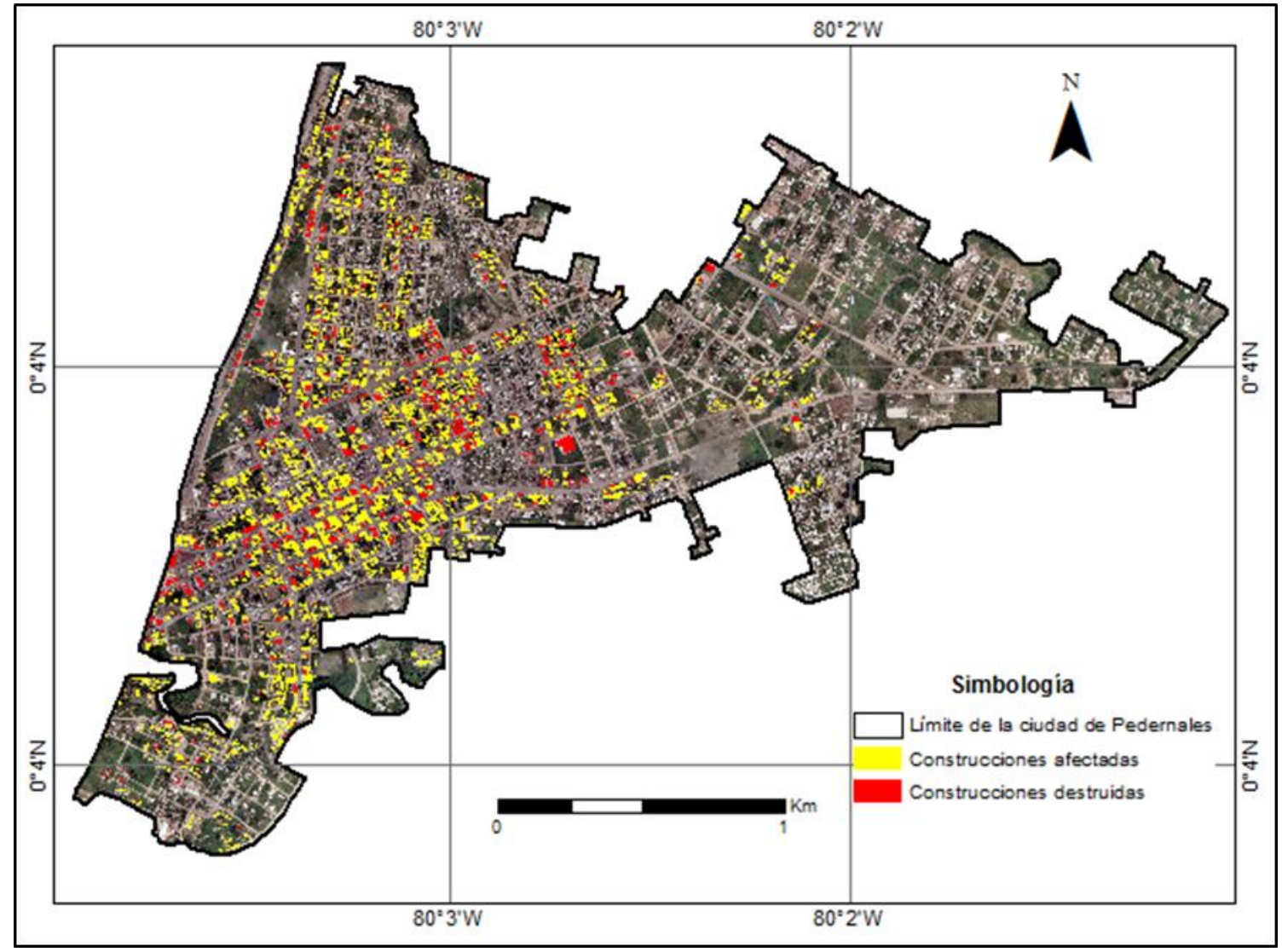

Fuente: Elaboración propia de los autores

\section{Discusión}

Al analizar la AFC de la ciudad de Pedernales y compararla con la infraestructura que presentan alguna avería o destrucción, se observa que un bajo porcentaje (6.89\%) de las edificaciones están asentadas en zonas con inadecuadas características físicas (lugares no aptos para este fin), y que la mayor cantidad de daños (52.10\%) se concentra en espacios aptos para la construcción (Figura 5). Estos resultados informan que la gran cuantía de infraestructura habitacional dañada o afectada durante el terremoto es consecuencia de los procesos constructivos puesto que el mayor impacto se localiza en terrenos con aptitudes para acoger asentamientos humanos. De aquí se desprende la indispensable y rigurosa labor de control que deben efectuar los GAD ante el desarrollo urbanístico (viviendas, escuelas, hospitales, vías de comunicación, infraestructura en general) con el objeto de mitigar el impacto negativo al que está sometida la población ante cualquier desastre natural; por ello, Metzger y Robert (2013) indican que las condiciones 
precarias de las viviendas pueden constituir una amenaza para la salud de sus habitantes en función del nivel de vulnerabilidad que manifiesten en ese momento.

Figura 5. Número de viviendas afectadas o destruidas en el terremoto en función de la AFC en Pedernales

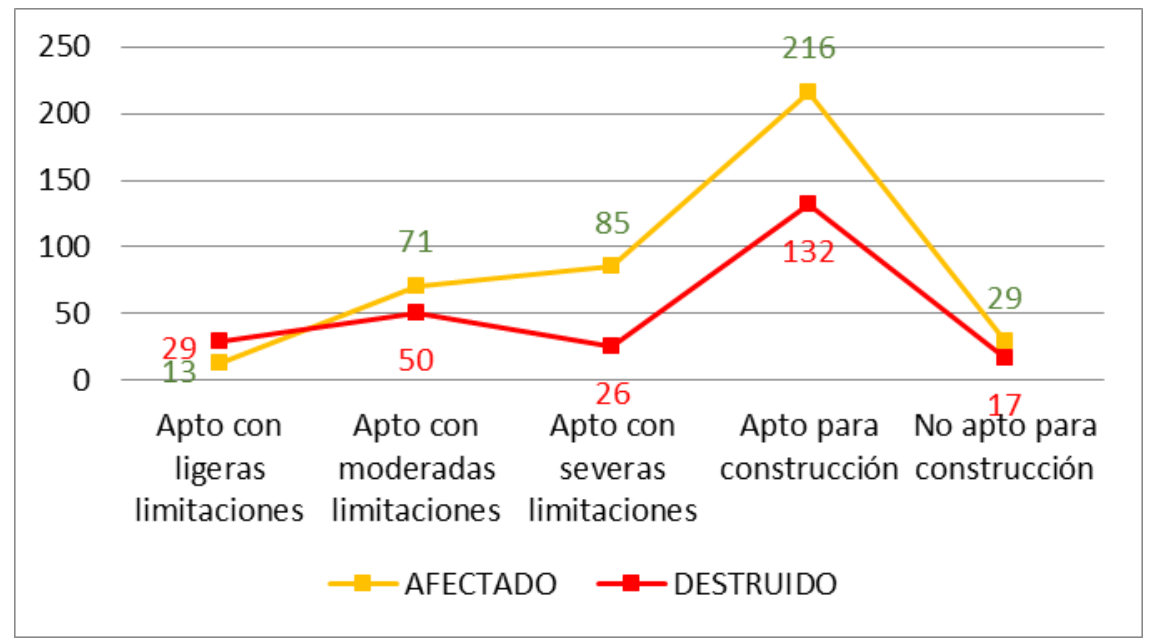

Fuente: Elaboración propia de los autores

En este sentido, si a las limitantes físicas del territorio se suman los incidentes de inundaciones, procesos de geodinámica externa e interna, se amplifican las variables a considerar dentro del análisis de AFC a fin de obtener un estudio más robusto que involucre este tipo de amenazas (Smith y Romero, 2009).

Por lo anterior, el presente modelamiento puede ser alimentado o retroalimentado al incluir nuevas y más variables que la ciencia y tecnología lo permitan: a mayor detalle, se pueden agregar parámetros geofísicos, geotécnicos y demás estudios que pulan y delimiten con mayor precisión la efectividad para el desarrollo de complejos habitacionales y del crecimiento urbano. Por lo anterior, la cartografía generada de Aptitud Física Constructiva (AFC) debe ser entendida como insumo de aporte para el ordenamiento territorial y no considerarse como un mapa que revele apertura para la edificación de viviendas sin estudios geotécnicos, por ejemplo, o, peor aún, sin apegarse a las normas vigentes respectivas (Norma Ecuatoriana de la Construcción NECSE-GM, 2014).

\section{Conclusiones}


Con la información geo-espacial de relieve, suelos, pendientes y litología, más la evaluación multicriterio, y los métodos de análisis jerárquicos, permitieron la categorización de unidades o espacios homogéneos para la compatibilidad e incompatibilidad constructiva para edificaciones. Se determinó cinco niveles de $\mathrm{AFC}$ en el territorio en estudio (Pedernales): apto para construcción, apto con ligeras limitaciones, apto con moderadas limitaciones, apto con severas limitaciones y no apto para construcción, donde los dos últimos ocupan el mayor porcentaje del territorio.

Pese a que gran parte de los asentamientos urbanos se encontraban en un área apta para la construcción no se descarta que se encuentren libres de una amenaza, como el caso del terremoto en el que se evidenció un alto porcentaje de edificaciones afectadas y destruidas relacionadas directamente con una inadecuada construcción.

Las directrices deben estar encaminadas en las formas y métodos constructivos y, preferentemente, en las áreas con mayor aptitud física del territorio para soportar tejidos urbanos y orientar a un mejor crecimiento de las ciudades objeto de este estudio.

\section{Referencias Bibliográficas}

Álvarez, M.; Moreno, A. y Mataix, C. (2013). The analytic hierarchy process to support decisionmaking processes in infrastructure projects with social impact, Total Quality Management \& Business Excellence, 24:5-6, 596-606. DOI: 10.1080/14783363.2012.669561

BBC Mundo (2016). Obtenido de Terremoto de magnitud 7,8 en la zona costera de Ecuador deja más de 600 muertos. Obtenido en: http://www.bbc.com/mundo/noticias/2016/04/160416_ecuador_terremoto_magnitud_colombia_p eru_bm

Cardona, O. D. (2008). Medición de la gestión del riesgo en América Latina. Revista Internacional de sostenibilidad, tecnología y humanismo, (3), 1-20.

Código Orgánico de Organización Territorial, Autonomía y Descentralización (COOTAD) (2015). Art. 140. Capítulo IV Del Ejercicio de las Competencias Constitucionales. Quito, Ecuador. Obtenido en: http://www.defensa.gob.ec/wpcontent/uploads/downloads/2016/01/dic15_codigo-organico-de-organizacion-territorialcootad.pdf 
Código Ecuatoriano de la Construcción (2002). Peligro sísmico, espectros de diseño y requisitos mínimos de cálculo para diseño sismo-resistente. Quito, Ecuador. Obtenido en: http://ficm.uta.edu.ec/descargas/codigo_ecuatoriano_construccion.pdf

Contreras, Y. D. C., y Beltrán, M. (2015). Reconstruir con capacidad de resiliencia: El casco histórico de la ciudad de Constitución y el sitio del desastre del terremoto y tsunami del 27 de febrero 2010. Revista Invi, 30(83), 79-115. Obtenido en: https://scielo.conicyt.cl/scielo.php?pid=S0718-83582015000100003yscript=sci_arttext

Emergency Events Data base (EM-DAT) (2012). Disaster profiles. Obtenido en: http://www.emdat.be/disaster-profiles

Gao, P.P.; Li, Y.P.; Sun, J. yLi, H.W. (2018). Coupling fuzzy multiple attribute decision-making with analytic hierarchy process to evaluate urban ecological security: A case study of Guangzhou, China. Ecological Complexity 34 (2018) 23-34. Elsevier Science. Available: https://doi.org/10.1016/j.ecocom.2018.03.001

Glavovic, BC, y Smith, GP (Eds.). (2014). Adaptarse al cambio climático: lecciones de la planificación de peligros naturales. Springer Sciencey Business.

Gómez Orea, D. (2002). Ordenación territorial. Madrid: Mundi-Prensa.

Instituto Nacional Estadísticas y Censos (INEC) (2010). Censo de Población, 2010. Quito, Ecuador. Obtenido en: http://www.ecuadorencifras.gob.ec/institucional/home/

Instituto Geofísico Escuela Politécnica Nacional (IGPN) (2016). Evaluación de intensidades, sismo en Pedernales. Informe Sísmico Especial No. 12. Quito, Ecuador. Obtenido en: http://igepn.edu.ec/servicios/noticias/1316-informe-sismico-especial-n-12-2016

Jumbo, J. K. (2018). Generación sobre la base SIG del mapa geomorfológico, escala 1: 25 000, orientado a la gestión territorial del cantón Balsas, provincia de El Oro (Bachelor'sthesis, Loja: Universidad Nacional de Loja). Recuperado de http://dspace.unl.edu.ec/handle/123456789/19791 Ley Orgánica de Ordenamiento Territorial, Uso y Gestión de Suelo (LOOTUGS) (2017). Título I. Principios y Reglas Generales. Objeto y Ámbito. Quito, Ecuador. Obtenido en: http://www.amevirtual.gob.ec/wp-content/uploads/2017/04/04-LEY-ORG\%C3\%81NICA-DEORDENAMIENTO-TERRITORIAL-USO-Y-GESTION-DE-SUELO-.pdf Metzger, P., y Robert, J. (2013). Elementos de reflexión sobre la resistencia urbana: usos criticables y aportes potenciales. Territorios. (28), 21-40. Obtenido en: http://revistas.urosario.edu.co/index.php/territorios/article/view/2550 
Norma Ecuatoriana de la Construcción (NEC-SE-GM) (2014). Geotecnia y diseño de cimentaciones. Acuerdo ministerial 0028. 19 de agosto de 2014:18-19. Obtenido en: https://www.habitatyvivienda.gob.ec/norma-ecuatoriana-de-la-construccion/

Quinceno, C. (2005) Escenarios de una catástrofe. Revista de antropología Iberoamericana AIBR (39). Obtenido en: http://www.aibr.org/antropologia/39ene/cronicas/ene0501.php.

Rodríguez, K. (2017). Urbanismo y procesos geodinámicos en la ciudad de Esmeraldas, Ecuador. Revista de Ciencias de Seguridad y Defensa, 2 (3): 17-24

Saaty, T.L. y Vargas, L.G. (2013). Decision Making with the Analytic Network Process, International Series in Operations Research y Management Science 195. Springer Science+Business Media New York. DOI: 10.1007/978-1-4614-7279-7_15

Smith, P. y Romero, H. (2009). Efectos del crecimiento urbano del Área Metropolitana de Concepción sobre los humedales de Rocuant-Andalién, Los Batros y Lenga. Revista de Geografía Norte Grande, (43), 81-93.

The United Nations Office for Disaster Risk Reduction (UNISDR) (2012). Disaster statistics. Obtenido en: http://www.unisdr.org/we/inform/disaster-statistics

Yuen, K.K.F. (2013). The Primitive Cognitive Network Process in healthcare and medical decision making: Comparisons with the Analytic Hierarchy Process, Appl. Soft Comput. J. Available: http://dx.doi.org/10.1016/j.asoc.2013.06.028

Zhang, Q.; Tan, W. ySun, Y. (2015). Parallel computing method for complex emergency systems based on multi-objective decision-making with analytic hierarchy process. 19th International Conference on Computer Supported Cooperative Work in Design (CSCWD) - Calabria, Italy. ISBN 978-1-4799-2002-0. 570-575 p.

Zinck, J.A. (2014). Geopedología: Elementos de geomorfología para estudios de suelos y riesgos naturales. Enschede, The Netherlands, ITC Faculty of Geo-Information Science and Earth Observation, p. 131

Zinck, J.A.; Metternicht, G.; Bocco, G. y Del Valle, H.F. (eds.) (2016). Geopedology: An Integration of Geomorphology and Pedology for Soil and Landscape Studies. Springer International Publishing. ISBN 978-3-319-19158-4,978-3-319-19159-1. DOI 10.1007/978-3-31919159-1 